\title{
On nonhomogeneous slip boundary conditions for 2D incompressible fluid flows
}

\author{
Paweł Konieczny \& Piotr Bogusław Mucha ${ }^{1}$ \\ Institute of Applied Mathematics and Mechanics \\ Warsaw University \\ ul. Banacha 2, 02-097 Warszawa, Poland \\ E-mail(PK): konieczny@hydra.mimuw.edu.pl \\ E-mail(PBM): mucha@hydra.mimuw.edu.pl
}

\begin{abstract}
The paper investigates the issue of existence of solutions to the stationary Navier-Stokes equations in a two dimensional bounded domain. The system is studied with nonhomogeneous slip boundary conditions admitting flow across the boundary. The main result proves the existence of weak solutions for arbitrary data. An advantage of our approach is that the proof is constructive. Properties of the obtained result allow to study turbulent flows in description of such phenomena as polymers and blood motion.
\end{abstract}

MSC: 35Q30, 75D05

Key words: the Navier-Stokes equations, slip boundary conditions, nonhomogeneous boundary data, large data

\section{Introduction}

Nonhomogeneous boundary conditions appear in numerous models in hydrodynamics. Thanks to them we are able to study phenomena with large velocity of fluids in bounded domains. Such systems are in particular interest of applied sciences [5],[6], because they can describe turbulent flows. Basic models in this area are based on the classical Navier-Stokes equations $[7],[12],[18]$. Sometimes there are coupled with extra equations analyzing additional information about the system, however mathematical and computational difficulties are connected with the description of motion of the fluid.

\footnotetext{
${ }^{1}$ corresponding author
} 
Mathematical aspects of these models deliver still a lot of interesting questions. The simplest approach is to reduce the nonhomogeneous system to a model with homogeneous boundary data. Then the tools of functional analysis can be applied effectively in the reasonable class of models. However the technique works if we are able to find a suitable extension of boundary data to have a possibility to obtain a good a priori bound. Such a construction is not elementary, since the behavior of nonlinear terms in the modified equation as well as the boundary terms must be controlled [7].

In this note we investigate the steady Navier-Stokes equations in a two dimensional bounded domain with nonhomogeneous slip boundary conditions

$$
\begin{array}{lr}
v \cdot \nabla v-\operatorname{div} \mathbf{T}(v, p)=F & \text { in } \Omega, \\
\operatorname{div} v=0 & \text { in } \Omega, \\
n \cdot \mathbf{T}(v, p) \cdot \tau+f v \cdot \tau=b & \text { on } \partial \Omega, \\
n \cdot v=d & \text { on } \partial \Omega,
\end{array}
$$

where $v=\left(v^{1}, v^{2}\right)$ is the velocity function, $p$ - the pressure, $\mathbf{T}$ is the stress tensor of Newtonian fluids: $\mathbf{T}(v, p)=\nu \mathbf{D}(v)-p I d$, where $\nu$ - positive constant is the viscosity coefficient, $I d$ - the identity matrix and $\mathbf{D}(v)=\nabla v+(\nabla v)^{T}=$ $\left\{v_{, j}^{i}+v_{, i}^{j}\right\}_{i, j=1,2}$ is the deformation tensor; $F$ is the external force, $n$ and $\tau$ are the normal and tangent vectors to boundary $\partial \Omega ; f$ is the friction coefficient which should be nonnegative, but in general not constant, $b$ and $d$ are boundary data: $d$ describes the flow across $\partial \Omega$ and $b$ can represent some extra friction forces at the boundary. Additionally we assume that $\Omega$ is simply connected, then $(1.1)_{2}$ implies the following compatibility condition

$$
\int_{\partial \Omega} d d \sigma=0
$$

which must be satisfied by datum $d$ from $(1.1)_{4}$.

Slip boundary conditions [4],[9],[14],[16] have slightly different properties than the most popular Dirichlet data. Relation $(1.1)_{3}$ is just Newton's second law which governs the motion of particles at the boundary and $d$ describes the flows across the boundary. If friction $f \rightarrow \infty$ and $b, d \equiv 0$ then we get at the limit the no-slip Dirichlet relation $\left.u\right|_{\partial \Omega} \equiv 0$ and if $f \equiv 0$ the fluid reacts with surface $\partial \Omega$ as the perfect gas [3],[13], [11]. These types of boundary relations appear in model of motion of blood, polymers or liquid metals [6],[9]. The above phenomena are related with flows with large velocity vectors what requires to examine the mathematical problem with inhomogeneous boundary data. 
The aim of the present paper is to prove existence of solutions to system (1.1) for arbitrary data. Since our proof is based on explicite constructions a direct way to obtain to the numerical scheme for system (1.1) is given. A similar problem for the Dirichlet boundary conditions, where the velocity vector at the boundary is given, in simply connected domains has been solved years ago [1],[8],[10] by the standard energy method applied straightforwardly to equations $(1.1)_{1,2}$. Using the Hopf construction one can extend the boundary data by introducing a vector field $a: \Omega \rightarrow \mathbf{R}^{2}$, then the sought function is modified as follows

$$
v=u+a .
$$

As a result a new system for the unknown function $u$ is obtained, but with homogeneous boundary data. This construction guarantees that for any $\epsilon>0$ one is able to find a vector field $a$ such that

$$
\left|\int_{\Omega} u \cdot \nabla a u d x\right| \leq\left.\epsilon|| u\right|_{H_{0}^{1}(\Omega)} ^{2}=\epsilon \int_{\Omega}|\nabla u|^{2} d x
$$

provided that $u \in H_{0}^{1}(\Omega)$. Homogeneity of boundary conditions simplifies the considerations, since all boundary terms vanish.

In the present case of slip boundary conditions the situation is more complex. A direct application of the Hopf method to equations (1.1) does not work, because homogeneity of slip boundary conditions do not imply $\left.u\right|_{\partial \Omega} \equiv 0$; the tangent part is not controlled, so we are not able to estimate boundary terms coming from the nonlinear term. That is the main reason why we should look for a new approach. We restrict our attention only to the two dimensional case, since the full three dimensional system requires probably a different more advanced and complex methods. Basic calculations show that we meet similar difficulties as in the problem for the Dirichlet conditions for non simply connected domains - the famous flux problem [2].

Here we reformulate the original problem (1.1). Taking the vorticity of equation $(1.1)_{1}$ we get

$$
v \cdot \nabla \alpha-\nu \Delta \alpha=\operatorname{rot} F \quad \text { in } \Omega,
$$

where

$$
\alpha=\operatorname{rot} v=v_{, x_{1}}^{2}-v_{, x_{2}}^{1}
$$

is the vorticity of the velocity and it is a scalar function and the comma denotes the differentiation. Next, as in [13],[14] from the boundary relations 
$(1.1)_{3,4}$ we obtain the Dirichlet data on the vorticity:

$$
\alpha=(2 \chi-f / \nu) v \cdot \tau+b-2 d_{, s} \quad \text { on } \partial \Omega,
$$

where $\chi$ is the curvature of the boundary.

To close the reformulation a way to recover the velocity should be described. This information can be obtained from the following problem

$$
\begin{aligned}
& \operatorname{rot} v=\alpha \text { in } \Omega, \\
& \operatorname{div} v=0 \text { in } \Omega, \\
& n \cdot v=d \text { on } \partial \Omega .
\end{aligned}
$$

The coupled system (1.5)-(1.7) and (1.8) is an alternative version of the original problem (1.1) and from now we will investigate it instead of system (1.1). It is elementary to see that each of solutions to the coupled system defines a solution to the original problem because the kernel of operator in (1.8) is trivial. The last fact follows from the simply connectedness of domain $\Omega$.

Let us recall the standard notation we use: $H^{1}(\Omega)$ is the Sobolev space of functions $f \in L^{2}(\Omega)$ for which $\nabla f$ exists in a weak sense and $\nabla f \in L^{2}(\Omega)$ equipped with the norm $\|f\|_{H^{1}(\Omega)}^{2}=\int_{\Omega}|f|^{2}+|\nabla f|^{2} d x ;\left(H^{1}(\Omega)\right)^{*}$ states for the Banach space of all bounded linear functionals defined on $H^{1}(\Omega)$ for which the following norm is finite:

$$
\|F\|_{\left(H^{1}(\Omega)\right)^{*}}=\sup _{x \in H^{1}(\Omega):\|x\| \leq 1}|F(x)| .
$$

The $L_{\infty}(\partial \Omega)$ is the Banach space of all essentially bounded functions on $\partial \Omega ; H^{1 / 2}(\partial \Omega)$ is the space of all functions $g$ on $\partial \Omega$, for which there exists $G \in H^{1}(\Omega)$ such that $g \equiv G_{\mid \partial \Omega}$ in the trace sense; we also define

$$
\|g\|_{H^{1 / 2}(\partial \Omega)}^{2}=\inf _{G \in H^{1}(\Omega): G \mid \partial \Omega \equiv g}\|G\|_{H^{1}(\Omega)}^{2} .
$$

For another (equivalent) definition of the space $H^{1 / 2}(\Omega)$ see Appendix. Finally $H^{-1 / 2}(\partial \Omega)$ is the Banach space of all bounded linear functionals on $H^{1 / 2}(\partial \Omega)$ (with a norm similar to $\left(H^{1}(\Omega)\right)^{*}$ norm).

The main result of our note is the following.

Theorem 1. Let $\partial \Omega \in C^{2}, F \in\left(H^{1}(\Omega)\right)^{*}, f \in L_{\infty}(\partial \Omega), d \in H^{1 / 2}(\partial \Omega)$ and $b \in H^{-1 / 2}(\partial \Omega)$; then there exists at least one weak solution to problem (1.1) such that $v \in H^{1}(\Omega)$ and for this solution the following estimate is valid

$$
\|v\|_{H^{1}(\Omega)} \leq c\left(\|d\|_{H^{1 / 2}(\partial \Omega)},\|b\|_{H^{-1 / 2}(\partial \Omega)},\|F\|_{\left(H^{1}(\Omega)\right)^{*}}\right) .
$$


Our proof of the above result is based on analysis of the coupled system (1.5)-(1.8). A key element is an extension related with boundary datum $(1.1)_{4}$. Having a suitable extention of $d$ we are able to find the a priori bound. The same as in the case of the Dirichlet boundary conditions the main difficulty is to prove an inequality being a modification of (1.4). Then we apply the Hopf approach to the vorticity problem (1.5)-(1.7) and here the kernel of our note is hidden.

A similar result even for non-simply connected domains has been proved in [15], however for a restricted class of domains, only.

As an elementary corollary of Theorem 1 we obtain the following regularity result.

Corollary. Let $\partial \Omega \in C^{\infty}, F \in C^{\infty}(\bar{\Omega}) ; f, b, d \in C^{\infty}(\partial \Omega)$, then the weak solution given by Theorem 1 is in the $C^{\infty}(\bar{\Omega})$-class and represents the classical solution to system (1.1).

The paper is organized as follows. First we construct an extension of boundary data which satisfies an inequality of type (1.4). Next, we show the a priori bound. In section 4 we present a sketch of the proof of existence of weak solutions and finally in Appendix we prove an auxiliary results about the regularity of the extension.

Throughout the paper we try to use standard notations [12],[18].

\section{The extension}

In this section we construct an extension of boundary data to get homogeneity property at the boundary. The properties are described by the below lemma.

Lemma 2.1 Let $\Omega$ be bounded domain with $\partial \Omega \in C^{2}$. Given $d \in H^{1 / 2}(\partial \Omega)$ satisfying compatibility condition (1.2).

Then for any $\epsilon>0$ there exists vector field $v_{0}$ such that $v_{0} \in H^{1}(\Omega)$ and

$$
\begin{array}{ll}
\operatorname{div} v_{0}=0 & \text { in } \Omega, \\
v_{0} \cdot \vec{n}=d & \text { on } \partial \Omega
\end{array}
$$

and for every $\varphi \in \bar{H}_{0}^{2}(\Omega)=H^{2}(\Omega) \cap\left\{\left.\phi\right|_{\partial \Omega}=0\right\}$ the following inequality holds:

$$
\left|\int_{\Omega}\left(v_{0} \cdot \nabla \varphi\right)^{2} d x\right| \leq \epsilon\|\Delta \varphi\|_{L^{2}(\Omega)}^{2} .
$$


Moreover, the following estimate is valid

$$
\left\|v_{0}\right\|_{H^{1}(\Omega)} \leq C\|d\|_{H^{1 / 2}(\partial \Omega)} .
$$

Proof. Let $s:[0, L] \rightarrow \mathbb{R}^{2}$ be a normal parameterization of boundary $\partial \Omega$, i.e.

$$
s([0, L])=\partial \Omega, \quad s(0)=s(L)=x_{0} \in \partial \Omega, \quad \text { and } \quad s^{\prime}(t)=1
$$

for a fixed point $x_{0}$ and $L$ - the length of $\partial \Omega$. Next we introduce the following map $p:[0, L] \times[0, \zeta] \rightarrow \mathbb{R}^{2}$ such that

$$
p\left(t_{1}, t_{2}\right)=s\left(t_{1}\right)-t_{2} \vec{n}\left(s\left(t_{1}\right)\right),
$$

where $\vec{n}$ is the outer normal vector to boundary $\partial \Omega$. If $\zeta$ is small enough (comparing to curvature $\chi$ of boundary $\partial \Omega$ ), then the map is one-to-one and $p \in C^{1}$. Moreover

$$
\operatorname{dist}\left(p\left(t_{1}, t_{2}\right), \partial \Omega\right)=t_{2} .
$$

Using the definition we compute the gradient of map $p$ as follows

$$
p_{, t_{1}}=\left(1-t_{2} \chi\right) \vec{\tau}\left(s\left(t_{1}\right)\right), \quad p_{, t_{2}}=\vec{n}\left(s\left(t_{1}\right)\right) .
$$

Then we see that

$$
p_{, 1} \perp p_{, 2} \quad \text { and } \quad(\nabla p)^{-1}=\left(\frac{1}{1-t_{2} \chi} \vec{\tau}, \vec{n}\right)^{T} .
$$

We proceed the construction of the extension. Introduce a function $D:[0, L] \rightarrow \mathbb{R}$ such that $D \in H^{3 / 2}((0, L))$ and

$$
D(t)=\frac{L}{2 \pi} \int_{0}^{t} d(p(s, 0)) d s .
$$

Next, we define a function $\bar{D}: \mathcal{S}^{1} \rightarrow \mathbb{R}$ (where $\mathcal{S}^{1}$ is the unit circle) such that

$$
\bar{D}(r(x)) \equiv D(x) \quad \text { for } x \in[0, L] \quad \text { and } \quad\|\bar{D}\|_{H^{3 / 2}\left(\mathcal{S}^{1}\right)} \leq C\|D\|_{H^{3 / 2}(0, L)},
$$

where $r:[0, L] \rightarrow[0,2 \pi]$ is a simple parameterization: $r(s)=\frac{2 \pi}{L} s$. Let $E: \mathcal{S}^{1} \times \mathbb{R}^{+} \rightarrow \mathbb{R}$ be an extension of function $\bar{D}$ such that $E \in H_{(l o c)}^{2}\left(\mathcal{S}^{1} \times \mathbb{R}^{+}\right)$ 
(see Lemma 5.1 with $u_{0}=\bar{D}$ in Appendix). Now we take $\xi:[0, L] \times[0, \zeta] \rightarrow \mathbb{R}$ defined as follows:

$$
\xi\left(t_{1}, t_{2}\right)=E\left(r\left(t_{1}\right), t_{2}\right) \eta_{\epsilon}\left(t_{2}\right),
$$

where a smooth function $\eta_{\epsilon}(t)$ is defined as follows:

$$
\eta_{\epsilon}(t)= \begin{cases}1 & \text { for } t<\gamma^{2}(\epsilon), \\ \epsilon \ln \frac{\gamma(\epsilon)}{t} & \text { for } \gamma^{2}(\epsilon) \leq t<\gamma(\epsilon), \\ 0 & \text { for } t \geq \gamma^{2}(\epsilon),\end{cases}
$$

where $\gamma(\epsilon)=\exp \left(-\frac{1}{\epsilon}\right)$. Then $\xi \in H^{2}\left([0, L] \times \mathbb{R}^{+}\right)$.

For $\epsilon<\zeta$ we use the mapping $p:[0, L] \times[0, \zeta] \rightarrow \mathbb{R}^{2}$ to define $\xi$ on $\Omega$ :

$\xi(x)=\xi\left(p^{-1}(x)\right)$ for $x \in p([0, L] \times[0, \zeta])$ and $\xi(x)=0$ otherwise.

To avoid misunderstandings we will denote $\nabla_{t}$ as a gradient in $\left(t_{1}, t_{2}\right)$ coordinates, and $\nabla_{x}$ as a gradient in $\left(x_{1}, x_{2}\right)$ coordinates. Then we have:

$$
\nabla_{x} \xi \cdot \vec{\tau}=d \quad \text { on } \partial \Omega
$$

since $\nabla_{x} \xi \cdot \vec{\tau}=\nabla_{t} \xi \cdot(\nabla p)^{-1} \cdot \vec{\tau}=\nabla_{t} \xi \cdot[1,0]=\xi_{, t_{1}}=D^{\prime}(t) r^{\prime}(t)=d(p(t, 0))$.

Our sought field will be given as follows

$$
v_{0}=\nabla_{x}^{\perp} \xi \quad \text { in } \Omega .
$$

By the construction conditions (2.1) are satisfied.

Let us show inequality (2.2). Taking $\varphi \in \bar{H}_{0}^{2}(\Omega)$ we have

$$
\begin{aligned}
\int_{\Omega}\left(v_{0} \cdot \nabla \varphi\right)^{2} d x & =\int_{0}^{L} \int_{0}^{\zeta}\left(v_{0} \nabla \varphi\right)^{2}|J p| d t_{1} d t_{2} \\
& \leq C \int_{0}^{L} \int_{0}^{\zeta}\left(v_{0} \cdot p_{, 1} \nabla \varphi \cdot p_{, 1}\right)^{2}+\left(v_{0} \cdot p_{, 2} \nabla \varphi \cdot p_{, 2}\right)^{2} d t_{1} d t_{2} \\
& =I_{1}+I_{2} .
\end{aligned}
$$

Recalling (2.8) and (2.13) we calculate the first integral

$$
I_{1}=C \int_{0}^{L} \int_{0}^{\zeta}\left[\left(\nabla_{x} \xi \cdot\left(1-t_{2} \chi\right) p_{, 2}\right)\left(\nabla \varphi \cdot p_{, 1}\right)\right]^{2} d t_{1} d t_{2} .
$$

Introducing the following notation:

$$
f_{1}\left(t_{1}, t_{2}\right)=\left(1-t_{2} \chi\right)^{2} \nabla_{x} \xi \cdot p_{, 2}, \quad g_{1}\left(t_{1}, t_{2}\right)=\nabla \varphi \cdot \vec{\tau},
$$


we calculate

$$
\begin{aligned}
f_{1}\left(t_{1}, t_{2}\right) & =\left(1-t_{2} \chi\right)^{2} \nabla_{t} \xi \cdot(\nabla p)^{-1} \cdot p_{, 2}=\left(1-t_{2} \chi\right)^{2} \nabla_{t} \xi \cdot[0,1] \\
& =\left(1-t_{2} \chi\right)^{2} \xi_{, t_{2}}=\left(1-t_{2} \chi\right)^{2}\left(\frac{\partial E}{\partial t_{2}} \eta_{\epsilon}\left(t_{2}\right)+E\left(t_{1}, t_{2}\right) \eta_{\epsilon}^{\prime}\left(t_{2}\right)\right)
\end{aligned}
$$

and since $\left(1-t_{2} \chi\right)^{2}$ is bounded we rewrite integral $I_{1}$ as follows

$$
\begin{aligned}
I_{1} & =C \int_{0}^{L} \int_{0}^{\zeta}\left|f_{1} g_{1}\right|^{2} d t_{1} d t_{2} \\
& \leq C \int_{0}^{L} \int_{0}^{\zeta}\left|\frac{\partial E}{\partial t_{2}} \eta_{\epsilon}\left(t_{2}\right)\right|^{2}\left|g_{1}\right|^{2} d t_{1} d t_{2}+C \int_{0}^{L} \int_{0}^{\zeta}\left|E\left(t_{1}, t_{2}\right) \eta_{\epsilon}^{\prime}\left(t_{2}\right)\right|^{2}\left|g_{1}\right|^{2} d t_{1} d t_{2} \\
& =I_{11}+I_{12} .
\end{aligned}
$$

To estimate integral $I_{11}$ we recall that supp $\eta_{\epsilon} \subset[0, L] \times[0, \epsilon]$ and the fact $H^{1}((0, L) \times(0, \zeta)) \subset L_{\infty}\left(0, \zeta ; L_{4}(0, L)\right)$, then we get

$$
\begin{aligned}
I_{11} & \leq C \int_{0}^{L} \int_{0}^{\epsilon}\left|\frac{\partial E}{\partial t_{2}} \eta_{\epsilon}\left(t_{2}\right)\right|^{2}\left|g_{1}\right|^{2} d t_{1} d t_{2} \\
& \leq \epsilon C\|E\|_{H^{2}(\Omega)}^{2} \int_{\Omega}|\Delta \varphi|^{2} d x .
\end{aligned}
$$

To consider $I_{12}$ we apply the Hopf estimate (see [7],[11]): for each $u \in$ $H_{0}^{1}(\Omega)$ the following bound is valid

$$
\left\|\frac{u}{\delta}\right\|_{L^{2}(\Omega)} \leq C\|u\|_{H_{0}^{1}(\Omega)}
$$

where $\delta(x)=\operatorname{dist}(x, \partial \Omega)$ and constant $C$ does not depend on $u$. By (2.10) we see that

$$
\eta_{\epsilon}^{\prime}\left(t_{2}\right)=\frac{\epsilon}{t_{2}} \text { for } t_{2} \in\left[\gamma^{2}(\epsilon), \gamma(\epsilon)\right] \text { and } \quad \eta_{\epsilon}^{\prime}\left(t_{2}\right)=0 \text { otherwise. }
$$

Moreover from the definition $g_{1} \in H_{0}^{1}(\Omega)$, hence with the help of (2.15), recalling (2.14) we conclude

$$
I_{12} \leq \epsilon C\|E\|_{H^{2}(\Omega)}^{2} \int_{\Omega}|\Delta \varphi|^{2} d x
$$

since $\left\|g_{1}\right\|_{H_{0}^{1}(\Omega)} \leq C\|\Delta \phi\|_{L_{2}(\Omega)}$. The integral $I_{2}$ is estimated similarly to the integral $I_{11}$, choosing support of $\eta_{\epsilon}$ small enough. Lemma 2.1 is proved. 


\section{A priori estimate}

To construct the a priori bound there is a need of the Korn inequality to control the $H^{1}(\Omega)$-norm of the solutions (for the proof see [17])

Lemma 3.1 Let $\partial \Omega \in C^{2}, f \geq 0$, and $\Omega$ is not a disk (see Note below). Then there exists $K>0$ such that for

$$
u \in H^{1}(\Omega) \text { such that } \operatorname{div} u=0 \text { and } n \cdot u=0
$$

the following estimate is valid

$$
\nu \int_{\Omega} \mathbf{D}^{2}(u) d x \geq K\|u\|_{H^{1}(\Omega)}^{2} .
$$

Note: If $\Omega$ is a disk we additionally assume $f \not \equiv 0$. Then for $u$ as above the following estimate holds:

$$
\nu \int_{\Omega} \mathbf{D}^{2}(u) d x+\int_{\partial \Omega} f(u \cdot \tau)^{2} d \sigma \geq K\|u\|_{H^{1}(\Omega)}^{2},
$$

for proper constant $K>0$.

Reasoning in this section may be easily modified to include this case.

Next, we prove the a priori bound.

Lemma 3.2 Sufficiently smooth solution to problem (1.1) satisfies the following estimate

$$
\|v\|_{H^{1}(\Omega)} \leq D A T A
$$

where DATA depends on $\|F\|_{H^{1}(\Omega)^{*}}, \Omega, \nu,\|b\|_{H^{-1 / 2}(\partial \Omega)},\|d\|_{H^{1 / 2}(\partial \Omega)}$.

Proof. The first step is to modify the sought function $v$ by the vector field $v_{0}$ from Lemma 2.1 with $\epsilon \leq K / 2$. Then the solution to (1.8) is in the form:

$$
v=u+v_{0}
$$

The above identity implies that function $u$ satisfies the following problem

$$
\begin{array}{ll}
\operatorname{rot} u=\alpha-\operatorname{rot} v_{0} & \text { in } \Omega, \\
\operatorname{div} u=0 & \text { in } \Omega, \\
n \cdot u=0 & \text { on } \partial \Omega .
\end{array}
$$


Since domain $\Omega$ is simply connected we apply the Poincare lemma and conclude existence of a scalar function $\phi$ (so called stream function, defined uniquely up to a constant) such that

$$
u=\nabla^{\perp} \phi=\left(-\partial_{x_{2}} \phi, \partial_{x_{1}} \phi\right) .
$$

Moreover the boundary conditions imply the following relation

$$
0=n \cdot u=n \cdot \nabla^{\perp} \phi=\frac{d}{d s} \phi
$$

so $\phi$ is constant on $\partial \Omega$ and we choose zero at the boundary. Taking into account (3.4) and (3.5) system (3.3) reads

$$
\begin{array}{ll}
\Delta \phi=\alpha-\operatorname{rot} v_{0} & \text { in } \Omega, \\
\phi=0 & \text { on } \partial \Omega .
\end{array}
$$

Next, we analyze the vorticity equation (1.5). Multiplying (1.5) by $\phi$, integrating by parts we get

$$
\begin{aligned}
\nu \int_{\Omega} \alpha \Delta \phi d x-\nu & \int_{\partial \Omega} \alpha \frac{\partial \phi}{\partial n} d \sigma= \\
& =\int_{\Omega} u \cdot \nabla \phi \alpha d x+\int_{\Omega} v_{0} \cdot \nabla \phi \alpha d x+\int_{\Omega} F \cdot \nabla^{\perp} \phi d x .
\end{aligned}
$$

Since $\frac{\partial \phi}{\partial n}=u \cdot \tau$, by (1.7) and (3.4) we get

$$
\begin{aligned}
\nu \int_{\Omega}\left(\alpha^{2}-\alpha \operatorname{rot} v_{0}\right) d x-\nu \int_{\partial \Omega} \alpha(v & \left.-v_{0}\right) \cdot \tau d \sigma= \\
& =\int_{\Omega} v_{0} \cdot \nabla \phi \alpha d x+\int_{\Omega} F \cdot \nabla^{\perp} \phi d x .
\end{aligned}
$$

Using the following identity:

$$
\int_{\Omega} \alpha^{2} d x-\int_{\partial \Omega} \alpha(v \cdot \tau) d \sigma=\int_{\Omega} \mathbf{D}^{2}(v)^{2} d x+\int_{\partial \Omega}\left((v \cdot \tau)^{2} f-b(v \cdot \tau)\right) d \sigma
$$


one gets that the 1.h.s. of (3.7) equals:

$$
\begin{aligned}
(\text { l.h.s. of }(3.7))= & \nu \int_{\Omega} \mathbf{D}^{2}(v) d x-\nu \int_{\Omega} \alpha \operatorname{rot} v_{0} d x+\nu \int_{\partial \Omega} \alpha\left(v_{0} \cdot \tau\right) d \sigma \\
& +\nu \int_{\partial \Omega}\left[(v \cdot \tau)^{2} f-b(v \cdot \tau)\right] d \sigma \\
= & \nu \int_{\Omega} \mathbf{D}^{2}(v) d x-\nu \int_{\Omega} \alpha \operatorname{rot} v_{0} d x+ \\
& +\nu \int_{\partial \Omega}\left[(2 \chi-f / \nu) v \cdot \tau+b-2 d_{, s}\right]\left(v_{0} \cdot \tau\right) d \sigma \\
& +\nu \int_{\partial \Omega}\left[(v \cdot \tau)^{2} f-b(v \cdot \tau)\right] d \sigma \\
= & \nu \int_{\Omega}\left(\mathbf{D}^{2}(u)+2 \mathbf{D}(u): \mathbf{D}\left(v_{0}\right)+\mathbf{D}^{2}\left(v_{0}\right)\right) d x-\nu \int_{\Omega} \alpha \operatorname{rot} v_{0} d x+ \\
& +\nu \int_{\partial \Omega}\left[(2 \chi-f / \nu)\left(u+v_{0}\right) \cdot \tau+b-2 d_{, s}\right]\left(v_{0} \cdot \tau\right) d \sigma \\
& +\nu \int_{\partial \Omega}\left[\left(u+v_{0}\right) \cdot \tau\right)^{2} f-b\left(\left(u+v_{0}\right) \cdot \tau\right] d \sigma .
\end{aligned}
$$

By the Korn inequality - Lemma 3.1 - we get

$$
\begin{aligned}
(\text { l.h.s. }) \geq & K\|u\|_{H^{1}(\Omega)}^{2}+\nu \int_{\Omega}\left[-C\left(|\nabla u|\left|\nabla v_{0}\right|+\left|\nabla v_{0}\right|^{2}\right] d x-\nu \int_{\Omega} \alpha \operatorname{rot} v_{0} d x+\right. \\
& +\nu \int_{\partial \Omega}\left[(2 \chi-f / \nu)\left(u+v_{0}\right) \cdot \tau+b-2 d_{, s}\right]\left(v_{0} \cdot \tau\right) d \sigma \\
& +\nu \int_{\partial \Omega}\left[\left(u+v_{0}\right) \cdot \tau\right)^{2} f-b\left(\left(u+v_{0}\right) \cdot \tau\right] d \sigma
\end{aligned}
$$

Since $\alpha=\operatorname{rot}\left(v_{0}+u\right)$ and $f \geq 0$ we see that there is no term of second order (with respect to $\|u\|_{H^{1}(\Omega)}$ ) with negative coefficient, so to close the estimate we should only look at the r.h.s. of (3.7). Since $u \cdot \nabla \phi=0$ we are only interested in the term $\int_{\Omega} v_{0} \cdot \nabla \phi \alpha d x$. Using Schwarz inequality we get

$$
\begin{aligned}
\left|\int_{\Omega} v_{0} \cdot \nabla \phi \alpha d x\right| & \leq\left\|v_{0} \cdot \nabla \phi\right\|_{L^{2}(\Omega)}\|\alpha\|_{L^{2}(\Omega)} \\
& \leq\left\|v_{0} \cdot \nabla \phi\right\|_{L^{2}(\Omega)} C\left(\|\nabla u\|_{L^{2}(\Omega)}+\left\|\nabla v_{0}\right\|_{L^{2}(\Omega)}\right) .
\end{aligned}
$$


Imposing no restriction on the viscosity coefficient we require Lemma 2.1 with $\epsilon \leq K / 2$ to get the following inequality:

$$
\left|\int_{\Omega} v_{0} \cdot \nabla \phi \alpha d x\right| \leq \frac{K}{2}\|\nabla u\|_{H^{1}(\Omega)}^{2}+C\|\nabla u\|_{L^{2}(\Omega)}\left\|\nabla v_{0}\right\|_{L^{2}(\Omega)} .
$$

From (3.9) and (3.10) we can derive the following inequality:

$$
\begin{aligned}
\frac{K}{2}\|u\|_{H^{1}(\Omega)}^{2} \leq & \mid \nu \int_{\Omega} \alpha \operatorname{rot} v_{0} d x+C\|\nabla u\|_{L^{2}(\Omega)}\left\|\nabla v_{0}\right\|_{L^{2}(\Omega)}+\left\|\nabla v_{0}\right\|_{L^{2}(\Omega)}^{2} \\
& -\nu \int_{\partial \Omega}\left[(2 \chi-f / \nu)\left(u+v_{0}\right) \cdot \tau+b-2 d_{, s}\right]\left(v_{0} \cdot \tau\right) d \sigma \\
& +\nu \int_{\partial \Omega} b\left(\left(u+v_{0}\right) \cdot \tau\right) d \sigma+\int_{\Omega} F \cdot \nabla^{\perp} \phi d x \mid .
\end{aligned}
$$

We cannot apply directly any inequality on terms consisting $u$ because we know only that $\frac{\partial u}{\partial \vec{n}}=0$ on the boundary. However if we recall that $u=\nabla^{\perp} \phi$ we see that $\int_{\Omega}|\nabla u|^{2}=\int_{\Omega}\left|\nabla^{2} \phi\right|^{2}$. Moreover $\phi \equiv 0$ on the boundary, so the norm $\int_{\Omega}\left|\nabla^{2} \phi\right|^{2}$ is equivalent to norm $\|\phi\|_{H^{2}(\Omega)}^{2}$, hence all terms from (3.11) consisting of $u$ and derivatives of $u$ are estimated by $\left(\int_{\Omega}\left|\nabla^{2} \phi\right|^{2} d x\right)^{1 / 2}=$ $\left(\int_{\Omega}|\nabla u|^{2} d x\right)^{1 / 2}$.

The above considerations together with (3.11) justify the following inequality:

$$
\|u\|_{H^{1}(\Omega)} \leq C\left(\left\|v_{0}\right\|_{H^{1}(\Omega)},\left\|b, 2 d_{, s}\right\|_{L^{2}(\partial \Omega)},\|F\|_{\left(H^{1}(\Omega)\right)^{*}}\right) .
$$

Since $v=u+v_{0}$ and $\left\|v_{0}\right\|_{H^{1}(\Omega)} \leq C\|d\|_{H^{1 / 2}(\Omega)}$ we obtain (3.1). Lemma 3.2 is proved.

\section{Existence}

In this section we give a sketch of a proof of existence. First, let us recall the system of equations (equivalent to the original system (1.1)):

$$
\begin{aligned}
v \cdot \nabla \alpha-\nu \Delta \alpha & =\operatorname{rot} F & & \text { in } \Omega, \\
\alpha & =(2 \chi-f / \nu) v \cdot \tau+b-2 d_{, s} & & \text { on } \partial \Omega, \\
\operatorname{rot} v & =\alpha & & \text { in } \Omega, \\
\operatorname{div} v & =0 & & \text { in } \Omega, \\
n \cdot v & =d & & \text { on } \partial \Omega .
\end{aligned}
$$


For system (4.1) we introduce the following definition of weak solutions:

Definition 4.1 We say that $v \in H^{1}(\Omega)$ is a weak solution to problem (1.1) (or (4.1)) iff $v=\nabla^{\perp} \phi+v_{0}$, where $\phi \in \bar{H}_{0}^{2}(\Omega)$, vector $v_{0}$ is defined by Lemma 2.1 with $\epsilon \leq K / 2$ and the following identity holds

$$
\begin{gathered}
\int_{\Omega}\left(\nabla^{\perp} \varphi+v_{0}\right) \cdot \nabla \psi(\Delta \varphi+\bar{\alpha}) d x+\nu \int_{\Omega}(\Delta \varphi+\bar{\alpha}) \Delta \psi d x+ \\
-\nu \int_{\partial \Omega}(2 \chi-f / \nu)\left(\nabla^{\perp} \varphi+v_{0}\right) \cdot \tau \frac{\partial \psi}{\partial \vec{n}} d \sigma=\int_{\Omega} F \cdot \nabla^{\perp} \psi d x+\int_{\partial \Omega}\left(b-2 d_{, s}\right) \frac{\partial \psi}{\partial \vec{n}} d \sigma
\end{gathered}
$$

for any $\psi \in \bar{H}_{0}^{2}(\Omega)$.

To show existence we apply the standard technique - the Galerkin method. Since $\bar{H}_{0}^{2}(\Omega)$ is Hilbertian and separable we take a base $\left\{w_{i}\right\}_{i=1}^{\infty}$

$$
\bar{H}_{0}^{2}(\Omega)=\overline{\operatorname{span}\left\{w_{1}, w_{2}, \ldots, w_{n}, \ldots\right\}} \|^{\|\cdot\|_{H^{2}(\Omega)}} .
$$

Next, we introduce a finite dimensional subspace of $\bar{H}_{0}^{2}(\Omega)$ :

$$
V^{N}=\operatorname{span}\left\{w_{1}, \ldots, w_{N}\right\}
$$

We assume, without loss of generality, that $\left(w_{i}, w_{j}\right)_{V^{N}(\Omega)}=\delta_{i j}$, where $(\cdot, \cdot)_{V^{N}(\Omega)}$ is the inner product in $V^{N}(\Omega)$. An approximation of the sought function $\phi$ will be searched in the form:

$$
\varphi^{N}(x)=\sum_{j=1}^{N} c_{j}^{N} w_{j} \in V^{N} .
$$

To find coefficients $c_{j}^{N}$ we solve the following system

$$
\begin{aligned}
& -\int_{\Omega}\left(\nabla^{\perp} \varphi^{N}+v_{0}\right) \cdot \nabla w_{k}\left(\Delta \varphi^{N}+\bar{\alpha}\right) d x-\nu \int_{\Omega}\left(\Delta \varphi^{N}+\bar{\alpha}\right) \Delta w_{k} d x+ \\
+\nu & \int_{\partial \Omega}(2 \chi-f / \nu)\left(\nabla^{\perp} \varphi^{N}+v_{0}\right) \cdot \tau \frac{\partial w_{k}}{\partial \vec{n}} d \sigma=-\int_{\Omega} F \cdot \nabla^{\perp} w_{k} d x-\int_{\partial \Omega}\left(b-2 d_{, s}\right) \frac{\partial w_{k}}{\partial \vec{n}} d \sigma
\end{aligned}
$$

for $k=1, \ldots, N$. 
Standard methods show existence to (4.3) - see [7], [11] or [18]. Repeating the calculations from section 3 we obtain a uniform in $N$ bound

$$
\left\|\nabla^{2} \phi^{N}\right\|_{L_{2}(\Omega)} \leq C
$$

The weak compactness delivers a Cauchy subsequence such that

$\nabla^{2} \varphi^{i} \rightarrow \nabla^{2} \varphi^{*} \quad$ weakly in $L^{2}(\Omega), \quad \nabla \varphi^{i} \rightarrow \nabla \varphi^{*} \quad$ strongly in $L^{2}(\Omega),(4.4)$ where $\phi^{*} \in \bar{H}_{0}^{2}(\Omega)$ defines the sought solution fulfilling Definition 4.1 . Convergences stated by (4.4) are sufficient to pass to the limit in nonlinear terms in the system. Theorem 1 is proved.

\section{Appendix}

In Appendix we construct the extension which was necessary in section 2 (function $E$ ).

Lemma 5.1 Given the following problem:

$$
\begin{aligned}
\left(\frac{\partial^{2}}{\partial t^{2}}+\frac{\partial^{2}}{\partial x^{2}}\right)^{2} u & =0 \text { in } \mathcal{S}^{1} \times \mathbb{R}^{+}, \\
\frac{\partial u}{\partial t} & =0 \text { on } \mathcal{S}^{1} \times\{0\}, \\
u(x, 0) & =u_{0}(x) \text { for } x \in \mathcal{S}^{1}, \\
u & \rightarrow 0 \text { as } t \rightarrow \infty,
\end{aligned}
$$

where $u_{0} \in H^{3 / 2}\left(\mathcal{S}^{1}\right)$. There exists a solution $u \in H_{\text {loc }}^{2}\left(\mathcal{S}^{1} \times \mathbb{R}\right)$ to this problem, satisfying the following estimate

$$
\|u\|_{H^{2}\left(\mathcal{S}^{1} \times(0,1)\right)} \leq C\left\|u_{0}\right\|_{H^{3 / 2}\left(\mathcal{S}^{1}\right)} .
$$

Proof. We define $E: \mathbb{Z} \times[0,2 \pi] \rightarrow \mathbb{R}$ as follows:

$$
E(k, z)=\left\{\begin{array}{l}
\sin k z \text { for } k>0 \\
\cos k z \text { for } k \leq 0
\end{array}\right.
$$

We have $\partial_{z} E(k, z)=k E(-k, z)$ and $\overline{\operatorname{span}\left\{\{E(k, z)\}_{k \in \mathbb{Z}}\right\}} \|^{\|\cdot\|_{L^{2}\left(\mathcal{S}^{1}\right)}}=L_{2}\left(\mathcal{S}^{1}\right)$. We require $u: \mathcal{S}^{1} \times \mathbb{R}^{+} \rightarrow \mathbb{R}$ to be in the following form:

$$
u(x, t)=\sum_{k \in \mathbb{Z}} c_{k}(t) E(k, x),
$$


where $c_{k}(t)$ are sought functions with initial data given by

$$
u_{0}(x)=\sum_{k \in \mathbb{Z}} c_{k}(0) E(k, x)
$$

Since $u_{0}(x) \in H^{3 / 2}\left(\mathcal{S}^{1}\right)$ and $\{E(k, x)\}_{k \in \mathbb{Z}}$ is an orthogonal basis of $L_{2}\left(\mathcal{S}^{1}\right)$ we have:

$$
\sum_{k} c_{k}^{2}(0)\left(1+|k|^{2}\right)^{3 / 2} \leq C\left\|u_{0}\right\|_{H^{3 / 2}}^{2} .
$$

From $(5.1)_{1}$ and (5.4) we get ordinary differential equations for coefficients $c_{k}(t)$ :

$$
\left(\frac{\partial^{2}}{\partial t^{2}}-k^{2}\right)^{2} c_{k}(t)=0 \quad \text { for every } k \in \mathbb{Z}
$$

Taking into account the initial condition $(5.5)$ and $\left(5.1_{4}\right)$ we find a solution to $(5.7)$

$$
c_{k}(t)=(1+|k| t) c_{k}(0) e^{-|k| t} .
$$

Let us show that $u \in H^{2}\left(\mathcal{S}^{1} \times(0,1)\right)$. First

$$
\begin{aligned}
\|u\|_{L^{2}\left(\mathcal{S}^{1} \times(0,1)\right)}^{2} & =\int_{0}^{1} \int_{0}^{2 \pi} \sum_{k \in \mathbb{Z}}(1+|k| t)^{2} c_{k}^{2}(0) E^{2}(k, x) e^{-2|k| t} d x d t \\
& \leq C \sum_{k}\left(1+|k|^{3}\right) c_{k}^{2}(0) \leq C\left\|u_{0}\right\|_{H^{3 / 2}\left(\mathcal{S}^{1}\right)}^{2} .
\end{aligned}
$$

Next, we prove $u_{t t} \in L^{2}\left(\mathcal{S}^{1} \times(0,1)\right)$ :

$$
\begin{aligned}
\left\|u_{t t}\right\|_{L^{2}}^{2} & \leq C \int_{0}^{1} \sum_{k} c_{k}^{2}(0)\left(1+|k|^{6} t^{2}\right) e^{-2|k| t} d t \\
& \leq C \sum_{k}\left(1+|k|^{3}\right) c_{k}^{2}(0)=C\left\|u_{0}\right\|_{H^{3 / 2}\left(\mathcal{S}^{1}\right)}^{2} .
\end{aligned}
$$

Other derivatives can be estimated similarly.

Acknowledgment. The authors would like to thank Piotr Rybka for useful discussions. The paper has been supported by Polish KBN grant No. 1 P03A 02130. 


\section{References}

[1] C.J. Amick, Existence of Solutions to the Nonhomogeneous Steady Navier-Stokes Equations, Indiana Univ. Math. J. 33, 6 (1984), 817-830.

[2] W. Borchers, K. Pileckas, Note on the Flux Problem for Stationary Incompressible Navier-Stokes Equations in Domains with Multiply Connected Boundary, Acta Appl. Math. 37 (1994), 21-30.

[3] T. Clopeau, A. Mikelić, R. Robert, On the vanishing viscosity limit for the 2D incompressible Navier-Stokes equations with the friction type boundary conditions. Nonlinearity 11 (1998), no. 6, 1625-1636.

[4] Farwig, R.: Stationary solutions of compressible Navier-Stokes equations with slip boundary conditions, Comm. PDE 14, (1989) 1579-1606

[5] Fontelos, M.A.; Friedman, A.: Stationary Non-Newtonian Fluid Flows in Channelslike and Pipe-like Domains, Arch. Rational Mech. Anal. 151 (2000) 1-43

[6] H.Fujita, Remarks on the Stokes flow under slip and leak boundary conditions of friction type. Topics in mathematical fluid mechanics, 73-94, Quad. Mat., 10, 2002.

[7] G.P. Galdi, An Introduction to the Mathematical Theory of the Navier-Stokes Equations, Springer Tracts in Natural Philosophy, 1994.

[8] E. Hopf, Ein allgemeiner Endlichkeitssatz der Hydrodynamik, Math. Ann. 117 (1941), 764-775.

[9] Itoh, S.; Tanaka N.; Tani A.: The initial value problem for the Navier-Stokes equations with general slip boundary condition, Adv. Math. Sci. Appl. 4, (1994) 51-69

[10] L. V. Kapitanskii, K. I. Pileckas, On spaces of solenoidal vector fields and boundary value problems for the Navier-Stokes equations in domains with noncompact boundaries, Proc. Math. Inst. Steklov 159(2) (1994), 3-34.

[11] Konieczny, P., On a steady flow in three dimensional infinite pipe, Coll. Math. 104 (2006), no. 1, 33-56

[12] Ladyzhenskaya, O.A.: The Mathematical Theory of Viscous Incompressible Flow, Gordon and Breach, New York, 1966

[13] P.B. Mucha, On the inviscid limit of the Navier-Stokes equations for flows with large flux, Nonlinearity 16 (2003), 1715-1732.

[14] P.B. Mucha, The Navier-Stokes equations and the maximum principle, Int. Math. Res. Not. 2004, no. 67, 3585-3605.

[15] P.B. Mucha, Flux problem for a certain class of two-dimensional domains. Nonlinearity 18 (2005), no. 4, 1699-1704.

[16] P.B. Mucha, W. Sadowski, Long time behavior of a flow in infinite pipe conforming to slip boundary conditions. Math. Methods Appl. Sci. 28 (2005), no. 15, 1867-1880. 
[17] Solonnikov, V.A.; Scadilov, V.E.: On a boundary value problem for a stationary system of Navier-Stokes equations, Trudy Mat. Inst. Steklov. 125 (1973) 186-199

[18] Temam,R.: Navier-Stokes Equations. North Holland, Amsterdam (1977). 